\title{
A Study on the Factors of Interactive Scenario Design on Ecotourism for Active Ageing
}

\author{
Li-Shu Lu *, Yu-Fan Ho \\ Department and Graduate School of Digital Media Design, National Yunlin University of Science and Technology, Yunlin, 64002, Taiwan \\ *Corresponding Author: luls@yuntech.edu.tw
}

Copyright (C) 2015 Horizon Research Publishing All rights reserved.

\begin{abstract}
Experience ecotourism particularly could encourage active ageing groups with sensory experience, and easily help them reciprocally interact. This study invited four active aging persons and two professional tour-guiding staff to conduct "walk aloud" and "walk through" interviews in an ecological tourism destination "Taiwan Nantou Sun Link Sea Forest Recreation Area" suitable for the elderly's tourism according to the results of the first phase so as to further extract the problems and demands that the elderly face in ecotourism. The results show that: The factors forming the active aging groups' eco-tourism travel experience, including the external environment, physiological and psychological factors. However, to enhance the positive experience of the active aging groups' ecotourism when both physiological and environmental factors meet the conditions, external situation involvement is an important medium to enhance the elderly's satisfaction, including both emotion and story. The emotion part contains the experience of five senses, accompanying emotion and nostalgia experience; while the story part includes daily life stories, new knowledge obtaining and sharing mechanism. This study suggests that the results obtained from the above can be applied to shape the scenario design of future ecotourism field to help develop services and related technology products in order to enhance the elderly's joy and experience in ecotourism.
\end{abstract}

Keywords Interactive Scenario Design, Experience Design, Active Aging, Ecotourism

\section{Introduction}

Taiwan government has proposed people-oriented technology triggered intellectual life-related construction blueprints. Therefore, relevant research issues have been developed, such as the health promotion-oriented issues for the seniors[13]. The studies concerning population aging indicated that Taiwan will become a "super-aged society" in the future (after 2025). Therefore, Taiwan government, scholars and experts, and civil societies developed and amended "Population Policy White Paper" in July 2013. The content mentions that the policy promoting the elderly's participation in leisure activities should notice that, after the elderly's retirement, leisure activities will become a more important part in their life. It is important for them to properly use time to engage in social participation and various leisure activities. Being close to nature is positively beneficial to the physiological and psychological health of the elderly [15]. Therefore, the elderly will become the active aging group when experiencing the atmosphere of nature and forgetting their age due to happiness $[7,8,9,16]$. Moreover, properly using technologies can increase the experience and learning of ecotourism and further achieve the objective of making the active aging group forget their age due to happy learning. At present, due to the progress of technologies, experiential tourism has also started to attach importance to the concept of 3T, namely, Travel, Tourism, and Technology[1]. Some scholars suggest that interactive experience design can improve sense of identification and sense of belonging. In addition, interactive experience design can be increased during travel to enhance the competitiveness of tourism industry $[3,10,11,12]$. Therefore, when technologies are integrated with active aging group's ecotourism, people's perceptual cognition, emotional expression, and experiences of environmental atmosphere and use of technology seem to be more important, which is the so-called scenario modeling.

Some scholars suggest that scenario refers to a story itself [4]. Benyon (2002) suggests that scenario refers to People's use of Technology to engage in certain Activities in certain Contexts (abbreviated as "PACT") [2]. In the design of interactive systems, the application of scenario is quite common. Scenario design has been put into practice internationally for years. Reviewing the studies on scenario design methods can help designers face the challenges of facing design, solve the cognitive problems between them and users, and fully apply scenario design methods to product development process $[2,4,5]$. To enable technological products to gently assist the elderly in engaging in ecotourism, design research on scenario development and design can be conducted and provided as reference for the interactive design of future experience. 
Therefore, the answer to the question about how to maintain the health and happiness of active aging group is to enable them to participate in ecotourism activities, which is the best leisure activity in life for maintaining health and happiness [14]. Therefore, in order to enable active aging group to enjoy ecotourism and profoundly experience the feelings of happy learning in travel, this study intends to develop an edutaining and interesting interactive scenario which meets the objectives of obtaining ecological knowledge and experiencing leisure.

\section{Methods}

In order to develop an experiential scenario which meets the objective of experiencing leisure and edutainment, this study conducted a two-stage pre-survey from August 1to December 25, 2013: (1) stage of research field survey; (2) depth-of-field observation. This study used occult observation and non-structured interviews to perform a preliminary exploration on the environment and behaviors of various types of ecotourism in the fields of ecotourism. This study then used active aging group's participatory work to investigate relevant issues, and used retrospective interviews and Walk Aloud to collect the data on travel mentality of active aging group. The procedure of research as shown in Figure 1.

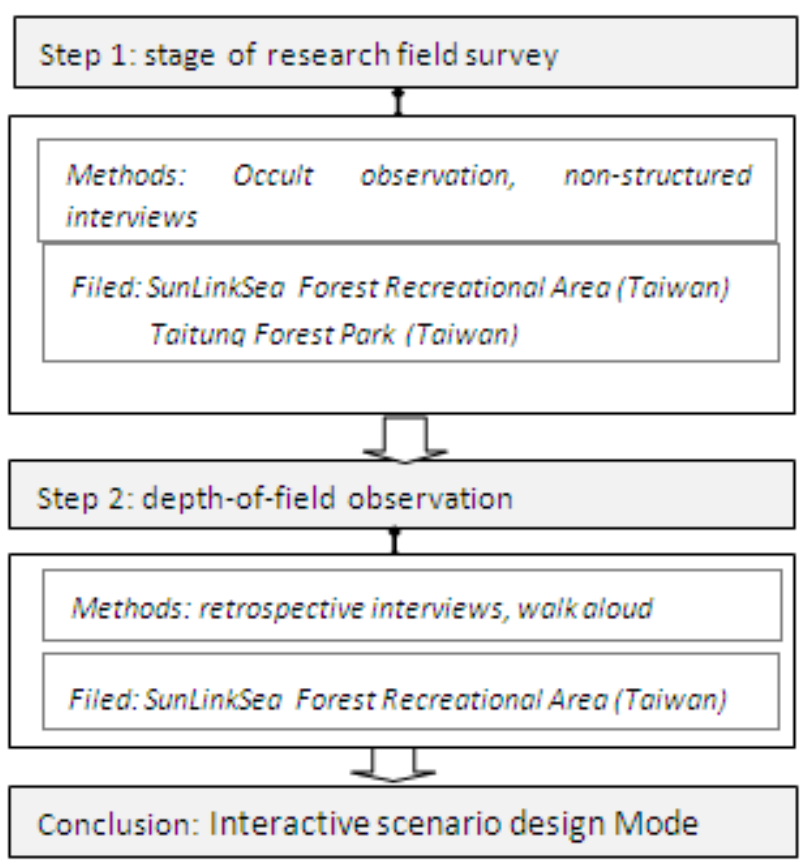

Figure 1. the procedure of research

\subsection{Field Survey and Preliminary Exploration on Behaviors}

This study preliminarily selected SunLinkSea Forest Recreational Area and Taitung Forest Park (as shown in Figure 2) in Taiwan as the sites of the stage 1 field survey and preliminary exploration $\mathrm{n}$ behaviors. This study used random sampling to select active aging group as the subjects. Occult observation and camera were used to record various behaviors of active aging group aged 55 and above who were able to engage in ecotourism activities. This study also used non-structured interviews to investigate the leisure feeling and perception of active aging group. The research tools included digital recorder, paper \& pen, and camera.

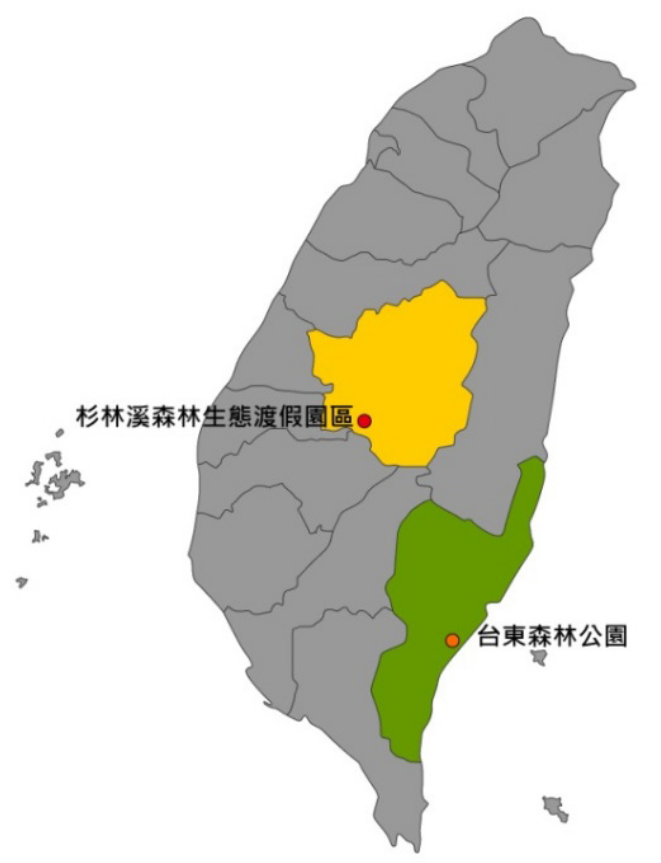

Figure 2. The research filds in Taiwan

\subsection{Confirmation of Field and In-depth Interviews}

This stage further selected Sun Link Sea Forest Recreational Area as the field for in-depth interviews. The reasons were: (1) Sun Link Sea is at medium altitude featured by specifies diversity; (2) Sun Link Sea Forest Recreational Area is the first private institution passing the certification of Environmental Education Facility of Environmental Protection Administration, Executive Yuan; (3) there is an ecological education center; (4) there is a complete docent service and training. There are diversified tourism types and abundant ecological species, including learning and leisure functions and a full range of passenger service equipment. Therefore, Sun Link Sea is a friendly field to active aging group. This study mainly used participatory workshop and field operation to better understand the potential needs of active aging group during ecotourism process. The subjects were divided into two groups for observation. Each group included one female subject of active aging, one male subject of active aging, one docent with professional knowledge, and two recorders. This study used retrospective interviews to investigate one group, and used walk aloud to investigate the other group. Cameras were used to record the full experience process of ecotourism, as shown in Figures 3-6. 


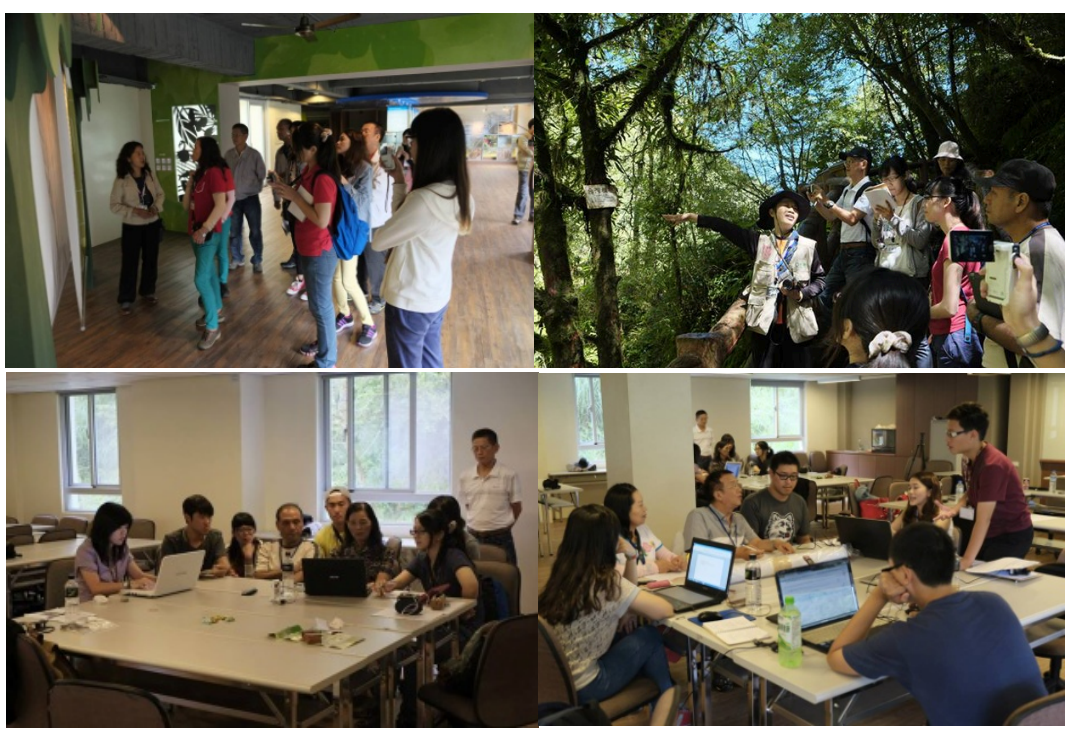

Figure 3, 4. walk aloud-a\&b; Figure 5, 6: interview-a\&b.

\section{Finding}

\subsection{Analysis on the Behaviors of Active Aging Group Participating in Ecotourism}

Most of the active aging group participates in tourism activities in the company of companions. Their companions may include spouse, families, and friends. There were also subjects in this study participating in ecotourism alone. However, the sample size was smaller. In order to compare the difference in subjects with/without companions and difference in number of subjects, this study divided the observed active aging group into single, double, and multiple people, as shown in Table 1.

The fields of ecotourism are usually located in mountains or natural environment. Owing to the limitations on time and manpower, this study could not comprehensively investigate various fields. Therefore, this study selected popular tourist attractions as the study field. The types of study fields are divided into: (1) trails/gallery roads: the roads where visitors can experience walking in mountain or nearby ocean. Trails and gallery roads are featured by natural and cultural resources or beautiful landscape where visitors can engage in various activities, such as ecotourism, natural experience, environmental education, leisure \& recreation, and landscape appreciation [6]; (2) gardens: gardens are indoor scenic spot where artificially cultivated featured flower species displayed and visitors can appreciate flowers for recreation; (3) designated landscape: featured landscape in the field where visitors can gather together to appreciate beautiful landscape.

During ecotourism experiencing process, active aging group will engage in a lot of interactive behaviors. Therefore, this study used AEIOU systemic approach to distinguish Activity from Interaction, as well as to define Activity as what Users do in the Environment and Interaction as the behaviors of exchange among Users, People and Objects. According to the results obtained from the observation and interviews at stage 1, this user-centered study divided active aging group into single, double, and multiple people travel. Moreover, this study also divided the fields into several types: trails/gallery roads, gardens, and designated landscape.

Table 1. Subjects described

\begin{tabular}{|c|c|c|c|c|}
\hline & & $\mathrm{S}$ & $\mathrm{T}$ & Appendix \\
\hline & Single & 1 & 3 & No other companion \\
\hline \multirow{4}{*}{$D$} & Couple & 7 & 4 & \\
\hline & Friends & $\mathrm{N}$ & 3 & \\
\hline & $\begin{array}{c}\text { Parents } \\
\text { /child }\end{array}$ & $\mathrm{N}$ & $\mathrm{N}$ & \\
\hline & $\begin{array}{l}\text { Grandparent } \\
\text { /grandchild }\end{array}$ & $\mathrm{N}$ & $\mathrm{N}$ & \\
\hline \multirow[t]{4}{*}{$M$} & Family & 6 & 7 & $\begin{array}{l}\text { Various combinations: } \\
\text { *Couple and child } \\
\text { *Couple and grandchild } \\
\text { *Couple, childe, and } \\
\text { grandchild } \\
\text { *Single man/woman and } \\
\text { child } \\
\text { *Single man/woman, child, } \\
\text { and grandchild } \\
\text { * Big family }\end{array}$ \\
\hline & Friends & 4 & 4 & $\begin{array}{c}\text { Various combinations: } \\
\text { *couple \& riend } \\
\text { *Couple, family, \& friend } \\
\text { *Friend group }\end{array}$ \\
\hline & Other group & $\mathrm{N}$ & $\mathrm{N}$ & \\
\hline & Total & 18 & 21 & \\
\hline
\end{tabular}

ps: S- SunLinkSea Forest Recreational Area; T- Taitung Forest Park; D-Double; M-Multiple

\subsection{Analysis on the Behaviors and Perception of Ecotourism}


Stage 2 depth-of-field observation found that, 4 behaviors would make active aging group feel delighted and further increase their experiential feelings during ecotourism experiencing, as shown in Figure 7: (1) nostalgia association with old memories; (5) five senses experiencing - experiencing five senses, hearing, touch, vision, taste, and smell can strengthen the memories; (3) sharing - the mutual sharing between companions can maintain and improve interpersonal relationship; (4) reward - increasing the sense of accomplishment of active aging group.

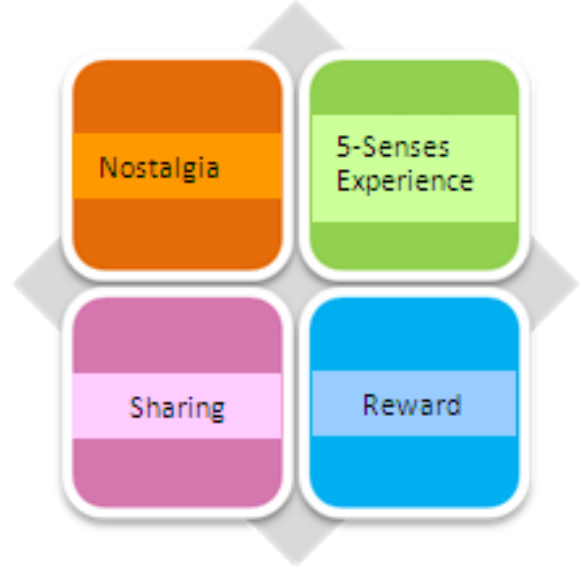

Figure 7. Analysis on the behaviors and perception of ecotourism

\section{Conclusions}

According to the preliminary exploration and analysis of stage 1 , the field of trails/gallery roads can provide diversified experiences. In addition, the guided tour provided by docents enables visitors to experience knowledge, completeness, and abundance. Therefore, the main schedule of participatory workshop of stage 2 was trails/gallery roads guided tour experiencing. In-depth travel of designated landscape can reduce the burden of active aging group: Designated ecotourism enables visitors to have sufficient time to fully experience ecotourism, as well as to better understand the knowledge and information of the field. Docents have to control adequate pace: The physical strength of active aging group is limited. Docents have to assess their physical condition to speed up or slow down the pace, as well as properly adjust the time of exposition and rest. The research conclusions and suggestions are as follows:

(1) Experiencing five sense can improve the quality of ecotourism: The singing of birds intrigued them. After docents introduced edible plants, the active aging group could strengthen the memories of knowledge about ecology by smelling (sense of smell) and eating (sense of taste). With the increase in age, the sensory functions will gradually decline. The use of other senses can make up the deficiency of single sense and increase the fun and impression of experiencing.

(2) Association of old memories and past experiences can strengthen the impression of experiences: When docents introduce species, if active aging group can associate them with old experiences or past memories, such as old songs, childhood food, they will be interested in them.

(3) Company of families and friends can increase active aging group's willingness to participate: The bonds between active aging group and their families and friends can be increased. They can share the same topics, and look after one another for safety concern.

(4) Stores or examples in daily life can increase the interest of active aging group: Association with daily life can strengthen the feelings of active aging group and further increase their interest. Stories tend to intrigue active aging group and make them intend to understand more.

(5) Unique and rare species or landscape will intrigue active aging group: In general, rare or unique species always can intrigue active aging group, increase their interest, and further make them intend to understand more.

(6) Sharing can improve the interpersonal relationship and memory storage of active aging group: Active aging group always intend to share with companions anything new or strange they have learned or seen to increase the interactions with companions.

Based on the above conclusions, this study summarized the scenario factors affecting the ecotourism experiences of active aging group, as shown in Figure 8.

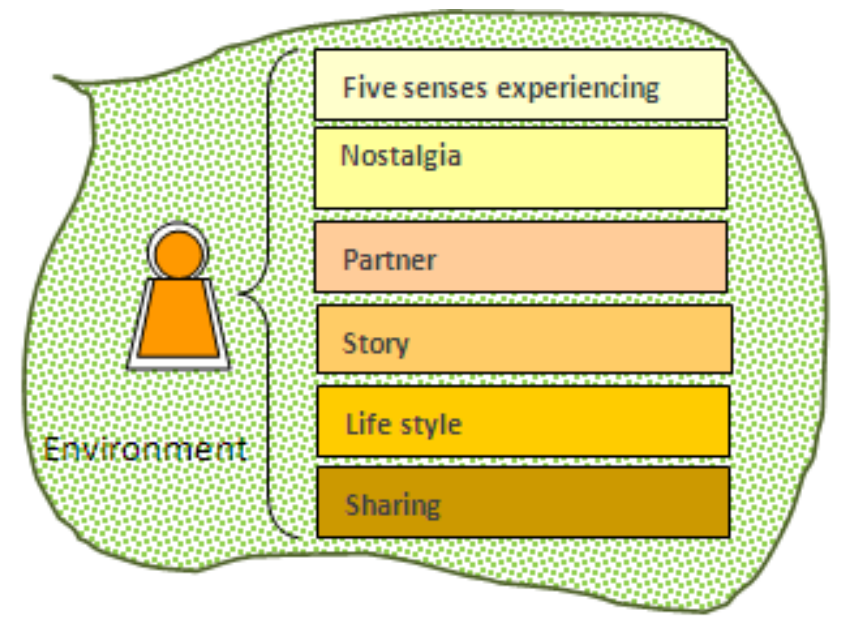

Figure 8. the scenario factors

\section{Acknowledgements}

This research is partially supported by the "102 Interactive scenario design on ecotourism for active ageing" of National Yunlin University of Science and Technology (College of Design), sponsored by the Ministry of Science and Technology, Taiwan, R.O.C under Grant no. NSC 102-2410-H-224 -033. 


\section{REFERENCES}

[1] Antonio Lopez de Ávila. (2011). World Tourism Organization (UNWTO) Affiliate Members AM-reports. Technology in Tourism, 1, 10.

[2] Benyon, D. \& Macauly, C. (2002). Scenario and the HCI-SE design problem. Interacting with Computers, 14, 397-405.

[3] Blanco. J., 2011, World Tourism Organization (UNWTO) Affiliate Members AM-reports -Technology in Tourism, Vol.1, p.6.

[4] Carrol, J. M. (2000). Making use: Scenario-based design of human-computer interaction. MIT Press.

[5] Darses, F. and Wolff, M. (2006). How do designers represent to themselves the users' needs? Applied Ergonomics, 37(6), 757-764.

[6] Forestry Bureau, Council of Agriculture, Executive Yuan, (2004). Retrieved from http:// http://www.forest.gov.tw/mp.asp?mp=1

[7] Frankl, V.E., \& Frank, V.E. (1970). The will to meaning: Foundations and applications of logotherapy. New American Library, New York.

[8] Kahn, P. H. (1997). Developmental psychology and the biophilia hypothesis: Children's affiliation with nature. Developmental review, 17(1), 1-61.

[9] Koenig, H. G. (1994). Aging and God: Spiritual pathways to mental health in midlife and later years. Haworth Pastoral Press/Haworth Press.

[10] Lindley, S.E., Harper, R., \& Sellen, A.(2008). Designing for elders: exploring the complexity of relationships in later life. Proceedings of the 22nd British HCI Group Annual Conference on People and Computers: Culture, Creativity, Interaction, 1.

[11] Nicola J. Bidwell Æ David Browning, 2010, Pursuing genius loci: interaction design and naturalplaces. Pers Ubiquit Comput., Vol. 14, pp.15-30.

[12] Tussyadiah, I. P., \& Fesenmaier, D. R. (2009). Mediating tourist experiences: Access to places via shared videos. Annals of Tourism Research, 36(1), 24-40.

[13] Ministry of the Interior (2013). Population Policy White Paper. 2013/12/22, from:

https://moe.senioredu.moe.gov.tw/ezcatfiles/b001/img/img/3 19/161755083.pdf

[14] Tseng, Y.L., Sato, N. (2012). A Study on the Process and Current Status of Japan's Promotion of Forest Healing Bases - A Case Study of Kyushu. Quarterly Journal of Forest Research, 34 (2), 161-171.

[15] Huang, H.L. (2012). The Richest "Active Aging Group" Appearing in Taiwan Three Years Later. CommonWealth Magazine. Retrieved on November 10, 2013, from: http://www.cw.com.tw/article/article.action?id=5033693.

[16] Yang, S.H. (2007). A Study on the Spiritual Status and Spiritual Education Need of the Elderly. Master's thesis, Graduate Institute of Education, National Kaohsiung Normal University. 